\title{
Nanoprobes for Multimodal Visualization of Bone Mineral Phase in Magnetic Resonance and Near-Infrared Optical Imaging
}

Pradeep P. Wyss, ${ }^{\dagger}$ Laura C. Herrera, ${ }^{\dagger}$ Nel S. Bouteghmes, ${ }^{\dagger, \dagger}$ Melika Sarem, ${ }^{\dagger, \S, \|}$ Wilfried Reichardt, ${ }^{\perp, \#}$ Jochen Leupold," Jürgen Hennig, and V. Prasad Shastri ${ }^{*}, \dagger, \S, \|$

${ }^{\dagger}$ Institute for Macromolecular Chemistry, University of Freiburg, Stefan Meier Strasse 31, 79104 Freiburg, Germany

${ }^{\ddagger}$ Université Pierre et Marie Curie, 4 Place Jussieu, 75005 Paris, France

${ }^{\S}$ Helmholtz Virtual Institute on Multifunctional Biomaterials for Medicine, Kantstrasse 55, 14513 Teltow, Germany

"BIOSS - Centre for Biological Signaling Studies, University of Freiburg, Schänzlestrasse 18, 79104 Freiburg, Germany

${ }^{\perp}$ German Cancer Research Center (DKFZ) and German Cancer Consortium (DKTK), Im Neuenheimer Feld 280, 69120

Heidelberg, Germany

${ }^{\#}$ Radiology-Medical Physics, University Medical Center Freiburg, Breisacher Strasse 60a, 79106 Freiburg, Germany

Supporting Information

ABSTRACT: Imaging agents with affinity for bone can enable early detection of changes to bone mineral density, which is a hallmark of many bone-associated pathologies such as Paget's disease and osteoporosis. Here, we report the development of a polymer nanoparticle (NP)-based multimodal imaging probe that enables visualization of bone mineral phase in nearinfrared (NIR) optical tomography and detection in $T_{2}$ weighted magnetic resonance imaging (MRI). Ultrasmall superparamagnetic iron oxide was first encapsulated in NPs derived by blending poly(DL-lactic-co-glycolic acid)-poly(ethylene glycol) (PLGA-PEG) with N-hydroxysuccinimide functionalized-PLGA (NHS-PLGA). Postmodification of NHS surface functionality on the NPs with alendronic acid (Aln), a bone-targeting ligand, yielded stable ultrasmall superparamagnetic iron oxide nanoparticles (USPIONs) containing NPs that exhibit good serum stability and favorable cytocompatibility. These post-Aln-modified NPs exhibit 8- to 10-fold higher affinity for synthetic and biogenic hydroxyapatite in comparison to NPs where Aln was introduced before NP formation and shorten the $T_{2}$ relaxation times in both agarose phantoms and fresh spongy bone, thus enabling the interrogation of bone mineral phase in $T_{2}$-MRI. Finally, by introducing an NIR-dye-modified PLGA during the NP formation step, NP probes that enable the visualization of bone mineral phase in both NIR optical tomography and MRI have been realized. The system presented herein meets many of the criteria for clinical translation and therefore opens new opportunities for bone imaging and targeted therapeutics.

\section{INTRODUCTION}

Functional imaging has become a mainstay in both preclinical and clinical studies. Among the imaging modalities, magnetic resonance imaging (MRI) has a wide acceptance because it employs nonionizing radiation and yields detailed structural information owing to its inherent high soft-tissue contrast. ${ }^{1}$ Between longitudinal $\left(T_{1}\right)$ - and transverse $\left(T_{2}\right)$-weighted MRI, $T_{2}$-imaging is particularly useful in visualization pathologies involving changes in water content, such as edema, and inflammation. Although qualitative approaches based on clustering of iron oxide nanoparticles (NPs) in response to matrix metalloproteases activity in vitro has been reported, ${ }^{2} T_{1}$ and $T_{2}$ MRI cannot yield quantitative information on biological processes. In this regard, fluorescence molecular tomography (FMT), an optical technique that uses near-infrared (NIR) light, which is weakly absorbed by the tissue and vasculature, allows for temporal assessment of quantitative changes to tissue function in live subjects ${ }^{3}$ and has emerged as a powerful imaging modality. ${ }^{4}$ FMT has been successfully used to visualize biological processes such as protease activity in atherosclerosis, ${ }^{5}$ tumor growth, ${ }^{3}$ and skeletal structures. ${ }^{6}$ Therefore, by combining MRI with FMT, fluorescence information can be assigned to body compartments, ${ }^{3,7}$ thereby providing information on biological processes.

Pathologies associated with bone such as Paget's disease and osteoporosis involve changes to bone mineral phase (BMP). Hydroxyapatite (HAp), the mineral phase of bone, is embedded in an organic collagen matrix. Bone undergoes constant remodeling via crosstalk between osteoclasts (boneresorbing cells) and osteoblasts (collagen- and mineraldepositing cells), ${ }^{8}$ with $5-10 \%$ of bone mass being renewed annually. ${ }^{9}$ Deregulation in this crosstalk can lead to accelerated

Received: June 21, 2016

Accepted: July 27, 2016

Published: August 8, 2016 


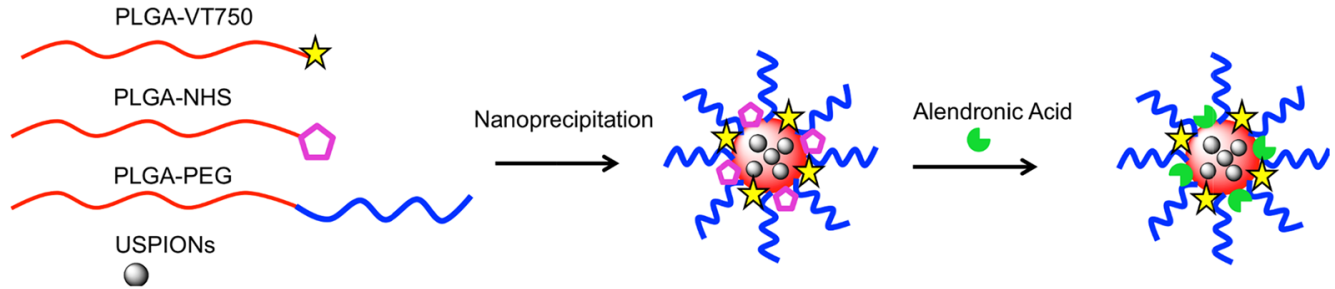

Figure 1. Schematic of the fabrication of multimodal NPs that can provide contrast in optical and MRI modalities. Note: VT750 is an NIR dye that is conjugated to PLGA.

Scheme 1. (A) Functionalization of PLGA with NHS and (B) Functionalization of PLGA-NHS with VT750 NIR Dye

A<smiles>[R]OC(C)C(=O)ON1C(=O)CCC1=O</smiles>

B<smiles>CC(C)OC(=O)C(C)ON1C(=O)CCC1=O</smiles>

bone loss as observed in osteoporosis and cancer metastasis to bone. ${ }^{10}$ Diagnosis of early indications of changes to BMP and bone mineral density (BMD) could help in early detection of bone pathologies. Therefore, imaging agents that bind to BMP and provide contrast in MRI and FMT can provide new insights and correlations between BMD and protease activity.

Toward the objective of realizing multimodal polymeric NPs with affinity to bone, we exploited the known affinity of alendronic acid (Aln), a bisphosphonate derivative to BMP. ${ }^{11}$ Targeting of anticancer drugs such as paclitaxel to bone using polymers, ${ }^{12}$ micelles, ${ }^{13}$ and dendrimers ${ }^{14}$ through conjugation with bisphosphonate is well established ${ }^{15}$ and has been explored for the treatment of Paget's disease and osteoporosis. $^{16}$ In the context of imaging, conjugation with bisphosphonate derivatives has also been exploited to target scintigraphy agents, fluorescent probes, radionuclide for positron emission tomography, ${ }^{17,18}$ and gadolinium chelates for $T_{1}$-weighted $\mathrm{MRI}$, which has been discussed in a recent exhaustive review by Cole et al. ${ }^{19}$

Because bone pathologies are accompanied by loss of the mineral phase, they are well suited for $T_{2}$-weighted $\mathrm{MRI}^{20}$ as changes in the mineral phase are expected to also change the water content in the bone. ${ }^{21}$ Therefore, bone-targeting agents, if properly designed, not only could serve as a targeted drug delivery system but also could exploit changes in the bone mineral content, to serve as a probe to enable early identification of these processes. For $T_{2}$-weighted MRI, ultrasmall superparamagnetic iron oxide nanoparticles (USPIONs) are the contrast agent of choice because they can disrupt the magnetic field within the tissue, providing dark contrast (negative contrast). ${ }^{22,23}$ Although the binding of $\gamma$ $\mathrm{Fe}_{2} \mathrm{O}_{3}$ NPs modified with a bisphosphonate derivative to synthetic hydroxyapatite has been reported, ${ }^{24}$ surprisingly, to date, no $T_{2}$-contrast agents capable of binding to BMP have been described. ${ }^{19}$

The use of USPIONs for imaging tissues can pose a few challenges. Free USPIONs are rapidly picked up by the cells of the reticuloendothelial system and show accumulation in the liver, which can lead to potential hepatic toxicity. ${ }^{25}$ However, this can be mitigated by altering the morphology of the $\mathrm{USPIONs}^{26}$ and encapsulating them within a polymer carrier. However, with respect to marrying USPIONs with bisphosphonate, the chelation of the phosphate groups with the iron in the USPIONs ${ }^{27}$ poses a severe limitation in encapsulating USPIONs using polymers conjugated to bisphosphonate.

In this study, we present a novel postmodification strategy to overcome this drawback, thereby synthesizing for the first time polymeric nanoprobes with high affinity to BMP, which also provide contrast in $T_{2}$-weighted $\mathrm{MRI}$ and the simultaneous visualization of BMP in NIR optical tomography. We achieved this by exploiting a blending approach to produce poly(DL-lactic acid-co-glycolic acid) (PLGA) NPs stabilized with polyethylene glycol (PEG) and having chemically accessible $N$-hydroxysuccinimide (NHS) groups on the surface, which upon postfunctionalization with Aln yielded NPs with a PEG- and Aln-rich surface. These PLGA-PEG/Aln NPs showed excellent solution stability, high binding capacity to $\mathrm{BMP}$, and $T_{2^{-}}$ contrast enhancement in MRI. By incorporating NIRfluorophore-labeled PLGA in the blending step, NPs with high affinity for BMP that can be imaged in MRI and optical modalities has been realized for the first time (Figure 1).

\section{RESULTS AND DISCUSSION}

NHS-PLGA Surface Functionalized NPs Containing USPIONs. Blending of PLGA with PLGA-PEG block copolymers to produce NPs with a PEGylated surface has been explored extensively. ${ }^{28,29}$ NPs bearing Aln on the surface have been prepared by blending PLGA-PEG with PLGAPEG-Aln and shown to accumulate in bone in a multiple myeloma model in mice. ${ }^{12}$ Because Aln has high chemical affinity for oxidized iron and therefore shows chelation towards USPIONs, it has been exploited to synthesize SPECT/MRI agents. ${ }^{17}$ We first verified the capacity of the USPIONs to 
interfere with NP formation using PLGA- $\mathrm{Aln}^{30}$ (see Materials and Methods for details of the synthesis) and found that the presence of USPIONs indeed disrupted NP formation in the nanoprecipitation method. ${ }^{31}$

The NPs produced in the presence of USPIONs are composed of large aggregates (size: $890 \pm 8 \mathrm{~nm}$, polydispersity index (PDI): 0.23 , Figure S1) and as such are too large for intravenous administration. We therefore postulated that postfunctionalization approach, wherein Aln is introduced for surface modification after the NP is formed, could enable the preparation of USPIONs containing NPs with bone-targeting Aln moieties. This approach would have an added advantage of allowing tunability of Aln density on the NP surface. We therefore end-functionalized PLGA with NHS (PLGA-NHS) as described in Scheme 1A and then blended PLGA-NHS with PLGA-PEG to yield NPs with a reactive NHS-rich surface. Furthermore, to confer visibility to the NPs in the NIR spectrum, PLGA modified with the NIR fluorescent dye VivoTag-750 (VT750) was synthesized starting from PLGANHS using a two-step synthesis as shown in Scheme $1 \mathrm{~B}$ and incorporated in the blending step.

The premise behind the incorporation of PLGA-PEG was to conform stability of the NPs via steric stabilization. However, a high concentration of PEG chains on the NP surface could diminish the accessibility and hence the reactivity of Aln to the NHS groups. Therefore, an optimization study was undertaken to identify the minimum weight-percentage of PLGA-PEG that would yield NPs with narrow polydispersity, in a size range that is suitable for intravenous administration while ensuring stability under serum conditions (Table S1). On the basis of this study, a blend composition of $80 \%$ PLGA-NHS, $15 \%$ PLGA-PEG, and 5\% PLGA-VT750 (total polymer concentration $5 \mathrm{mg} / \mathrm{mL}$ ) was deemed optimum because it yielded NPs below $150 \mathrm{~nm}$ with a PDI of less than 0.2 . The presence of a surface rich in NHS groups was confirmed by the charge inversion of the NP from negative $(\sim-24 \mathrm{mV})$ to positive $(\sim+8 \mathrm{mV})$ (Table 1$)$, and this is consistent with the $\mathrm{p} K_{\mathrm{a}}$ of

Table 1. Size and Zeta Potential of Nanoparticle Preparations

\begin{tabular}{|c|c|c|c|}
\hline NPs & $\underset{(\mathrm{nm})}{\text { size }} \underset{(\operatorname{average} \pm S D)}{ \pm}$ & PDI & $\begin{array}{c}\text { zeta potential } \\
(\text { average } \pm S D)(\mathrm{mV})\end{array}$ \\
\hline PLGA & $175 \pm 1$ & 0.16 & $-24.7 \pm 0.5$ \\
\hline PLGA-NHS & $148 \pm 3$ & 0.18 & $+8.2 \pm 0.7$ \\
\hline $\begin{array}{l}\text { blended NHS } \\
\text { functionalized NPs }\end{array}$ & $139 \pm 2$ & 0.16 & $+4.7 \pm 0.5$ \\
\hline
\end{tabular}

${ }^{a}$ PLGA-NHS/PLGA-PEG/PLGA-VT750 at 80/15/5 weight-percentage with USPIONs $0.1 \mathrm{mM}\left(23.1 \mu \mathrm{g} / 5 \mathrm{mg}\right.$ of PLGA) $\mathrm{Fe}_{3} \mathrm{O}_{4}$.

NHS, which is $7.8 .^{32}$ Because the size, PDI, and surface chemistry of the USPIONs can impact the encapsulation efficiency, USPIONs were synthesized by thermal decomposition of iron pentacarbonyl at high temperatures, which yielded particles with an average size of $5 \mathrm{~nm}$, a narrow PDI, and excellent magnetic properties. ${ }^{26}$ Furthermore, using this synthesis approach, the surface of the USPIONs is coated with oleylamine, ensuring that the particles are stable and dispersible in the polymer solution during the preparation of the NPs using the nanoprecipitation method. ${ }^{31}$ The NPs containing USPIONs were prepared using the blend system, and the uniform distribution of the USPIONs within the NPs was verified using transmission electron microscopy (TEM) (Figure 2A).
Postmodification of NHS-PLGA NPs Containing USPIONs with Alendronic Acid. NHS-PLGA NPs encapsulating USPIONs were first dialyzed against distilled water to remove residual organic solvents, and the NP suspension was postmodified by mixing with an aqueous Aln solution $(0.2 \mathrm{mg} / \mathrm{mL})$ before it was again dialyzed against water to remove unbound molecules (Scheme 2). Aln-sodium salt was synthesized by a previously published method $^{16}$ with minor modification, notably substituting $\mathrm{PCl}_{5}$ for $\mathrm{PCl}_{3}$, resulting in a $78 \%$ yield, and its structure was verified using IR, ${ }^{1} \mathrm{H}$ nuclear magnetic resonance (NMR), and ${ }^{31} \mathrm{P}$ NMR spectroscopy (Figure S2).

The zeta potential analysis of the NP suspensions post reaction revealed an inversion of the surface charge from positive to highly negative $(-32.2 \pm 1.7)$, which is consistent with the covalent linkage of Aln to the NP surface. Importantly, the modification step had minimal impact on the average size of the NPs, which remained relatively unchanged with a narrow PDI $(163 \pm 1 \mathrm{~nm}$, PDI $=0.16)$, suggesting that the encapsulated USPIONs did not interfere with the postmodification with Aln. Further evidence for the presence of Aln sodium salt was obtained by energy-dispersive X-ray (EDX) analysis of lyophilized NPs, where strong signals corresponding to phosphorous and sodium were observed in addition to iron from the encapsulated USPIONs (Figure 2B), whereas in the case of premodified control NPs (PLGANHS), no such peaks were detected (Figure S3). Additional evidence for the presence of Aln on the NP surface was gathered from the ${ }^{31} \mathrm{P}$ NMR spectra (Figure $2 \mathrm{C}$ ), where the presence of a lone phosphorous peak indicated that a single phosphorous-containing species was present on the NP, thus effectively excluding nonspecific chemisorption of Aln during the modification step. In comparison, when the PLGA-PEG/ PLGA NPs were incubated with Aln, no ${ }^{31} \mathrm{P}$ peak was detected, suggesting that Aln is not adsorbed through electrostatic interactions but is indeed covalently bound to the NP surface (Figure S4). The postfunctionalized Aln-PLGA NPs were found to be stable for 7 days even in serum containing cell media (Figure S5).

Dose-Dependent Binding of Aln-PLGA NPs to Synthetic and Biogenic HAp. PEG-PLGA NPs, NHSPLGA NPs, and Aln-PLGA NPs were incubated with HAp granules for $3 \mathrm{~h}$ and analyzed using a scanning electron microscope (SEM) (Figure 3A-C). While the PEG-PLGA NPs, as expected, did not bind to the surface, the NHS-PLGA NPs showed slight binding towards Hap, which may be attributed to the affinity between the slightly positively charged surface of the NHS-PLGA NPs and the negatively charged surface of the HAp granules. However, the Aln-PLGA NPs showed a remarkable binding capacity to the HAp surface with a complete coverage of the surface by the NPs (Figure 3C). To understand the binding behavior, a time course study was undertaken using Aln-PLGA NPs modified with VT750, and the NP binding was quantified using FMT. Total fluorescence associated with the NP stock solution was first quantified within an agarose phantom, and this value was used to determine the fraction of the adsorbed NPs. A representative FMT volumetric projection of the HAp granules treated with VT750-labeled Aln-PLGA NPs and then embedded in an agarose phantom is shown in Figure 3D. It was found that the adsorption of the Aln-PLGA NPs on the HAp surface showed first-order saturation kinetics with rapid adsorption in the first hour followed by saturation in $2-3 \mathrm{~h}$ (Figure $3 \mathrm{E}$ ), with a maximum 

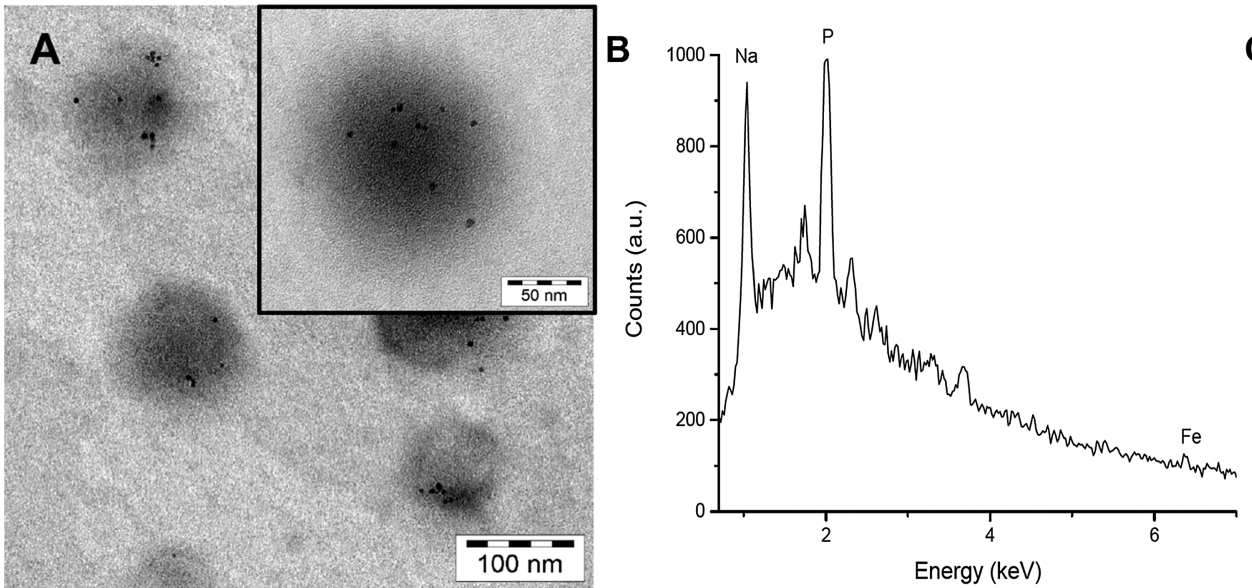

C

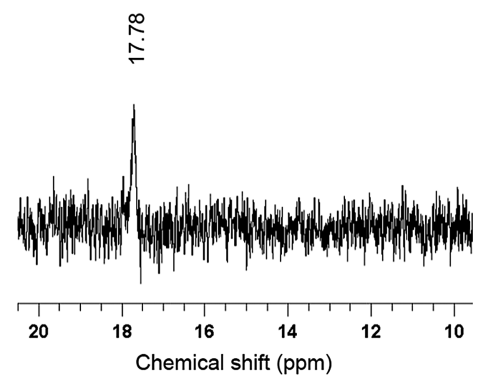

Figure 2. Characterization of freeze-dried Aln-PLGA NPs. (A) TEM image of the encapsulated USPIONs in Aln-PLGA NPs. (B) EDX spectra of the Aln-PLGA NPs. (C) ${ }^{31}$ P NMR spectra (12 800 scans) of Aln-PLGA NP suspensions.

Scheme 2. Surface Modification of NHS-PLGA NPs with Alendronic Acid

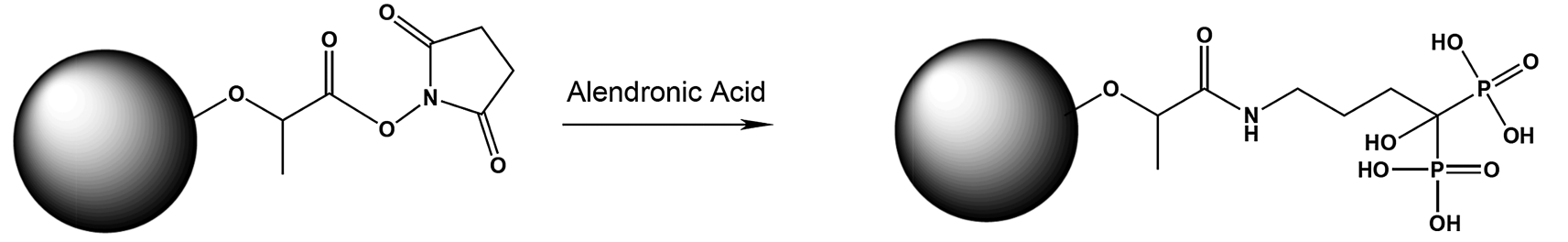

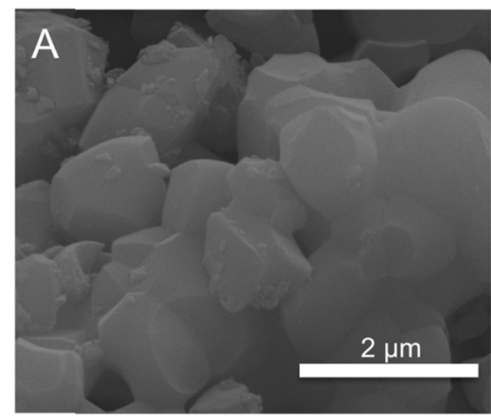

D

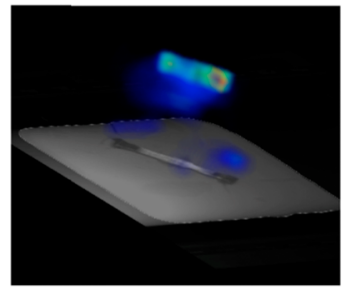

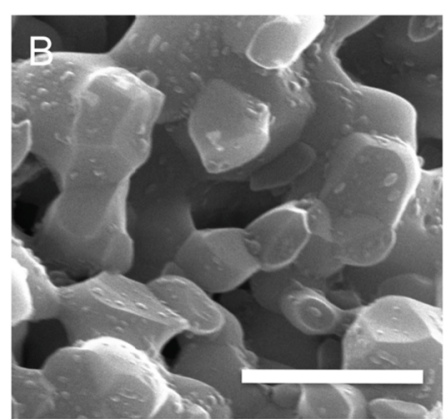

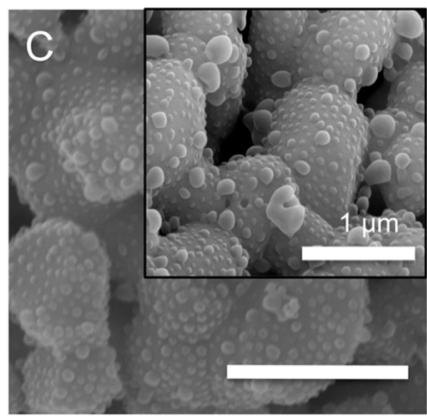

$\mathrm{F}$
E

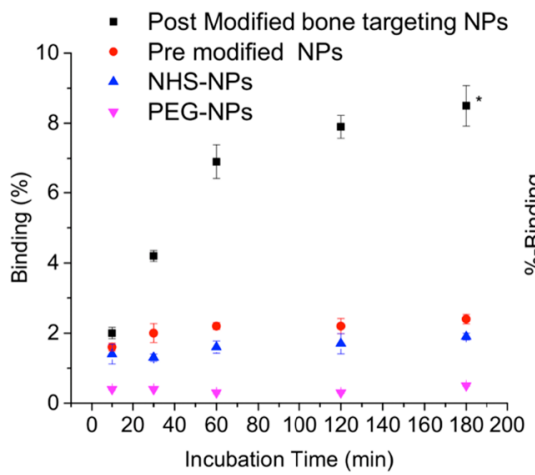

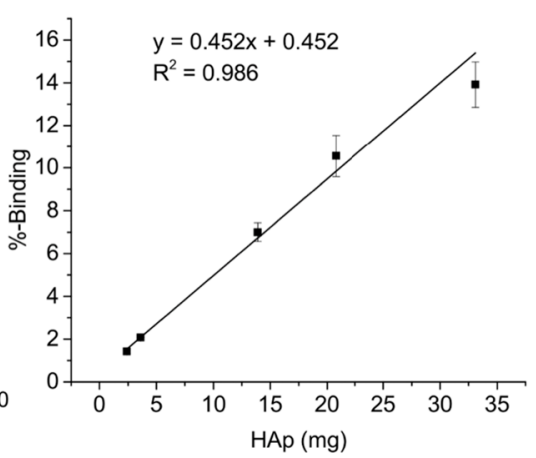

Figure 3. Visualization and quantification of Aln-PLGA NP adsorption on synthetic HAp granules. SEM images of the surface of HAp granules after incubation with NPs for $3 \mathrm{~h}$ (A) PEG-PLGA NPs, (B) NHS-PLGA NPs, and (C) Aln-PLGA NPs. (D) Representative reconstructed FMT volume of HAp in an agarose phantom after incubation with the Aln-PLGA NPs. (E) Adsorption behavior of the Aln-PLGA NPs $(n=3)$ (postmodified) versus NHS-PLGA $(n=3)$ PEG-PLGA $(n=3)$ (nonbinding controls) and PLGA NPs premodified with Aln (binding control, $n=$ 3). The asterisk indicates statistical significance between post- and premodified NPs with a $p$-value of 0.023. (F) Adsorption of Aln-PLGA NPs as a function of HAp mass.

of $\sim 8 \%$ of the NPs being adsorbed onto the HAp surface. In comparison, adsorption of PEG-PLGA and NHS-PLGA, both of which lack Aln, was less than $2 \%$ after 3 h. More importantly, the NPs produced using PLGA premodified with Aln showed 
poor binding behavior that was in the same range as the nonspecific controls (NHS-NPs and PEG-NPs). Furthermore, the binding of the Aln-PLGA NPs, that is postmodified, was fourfold greater than that of the premodified NPs after $3 \mathrm{~h}$; this difference was statistically significant $(p=0.023)$. This proved the validity of our approach that a postmodification strategy would yield superior outcomes. Furthermore, the Aln-PLGA NPs binding to the HAp surface was preserved in the cell culture medium supplemented with serum, which is physiologically more relevant (Figure S6). The above findings taken in their totality offer compelling evidence for the postmodification strategy presented herein. SEM analysis provided further qualitative evidence for the increased NP binding with longer incubation time (1-6 h) (Figure S7). The saturation kinetics suggested that the limiting factor in the adsorption of the AlnPLGA NPs onto the HAp surface was the availability of surface area. Because bone is highly porous, a linear scaling relationship between NP-associated fluorescence and surface area is necessary for qualitative analysis. Therefore, the Aln-VT750labelled Aln-PLGA NPs were incubated with an increasing amount of HAp granules, which corresponds to an increase in the surface area, and a linear correlation was observed up to a 10-fold increase in HAp mass (Figure 3F). This suggests that the system described herein can be used to quantify bone volume (mass).

Cytocompatibility and Binding Affinity of Aln-PLGA NPs toward Biogenic HAp. Cytocompatibility of AlnPLGA-VT-750 NPS. Toxicity of nanomaterials can be a limiting factor in clinical translation. We therefore investigated the cytocompatibility of the Aln-PLGA NPs toward osteoblasts because they are responsible for the mineralizing of mammalian skeletons. Osteoblasts were differentiated from human bone marrow-derived mesenchymal stem cells (MSCs) for 21 days, and their osteogenic lineage was confirmed by the upregulation of mRNA for type-1 collagen, alkaline phosphate, and bone sialoprotein using real-time quantitative polymerase chain reaction (RT-qPCR) on day 7 of differentiation and deposition of mineral phase on day 21 of differentiation (Figure S8). In addition to osteoblasts, toxicity toward Hepa 1-6, a mouse hepatoma-derived cell line that is routinely used for screening liver toxicity, ${ }^{26}$ and mouse macrophages (RAW 264.7) was undertaken. The reason for including macrophages in this screening was that NPs were cleared from circulation primarily by macrophages. Cells were incubated with the Aln-PLGA NPs at different concentrations, ranging from 31 to $500 \mu \mathrm{g} / \mathrm{mL}$ for $24 \mathrm{~h}$, and the cytocompatibility was assessed by the 3-(4,5dimethylthiazol-2-yl)-2,5-diphenyltetrazoliumbromid (MTT) assay. The Aln-PLGA NPs showed no appreciable toxicity in all three cell systems at concentrations below $125 \mu \mathrm{g} / \mathrm{mL}$, but a small decrease in cell viability was observed at 150 and $500 \mu \mathrm{g} /$ $\mathrm{mL}$ in all cell types (Figure 4). These data are consistent with the published reports on NP dose escalation and cell viability. ${ }^{26}$

Binding of Aln-PLGA-VT-750 to Biogenic HAp. Because the mineral deposits within biological environments were of much smaller dimension and possibly of different chemical composition than that of synthetic HAp, the ability of the AlnPLGA NPs to bind to mineral deposits secreted by osteoblasts was ascertained. Osteoblasts were differentiated from human MSCs for 21 days, and the secretion of BMP by the osteoblasts was verified by Alizarin Red staining (Figure S9). Osteoblasts were incubated with Aln-PLGA NPs and control NPs (premodified PLGA-Aln) for $2 \mathrm{~h}$ in the presence of serum, and the cells were incubated with Quin-2, which is an
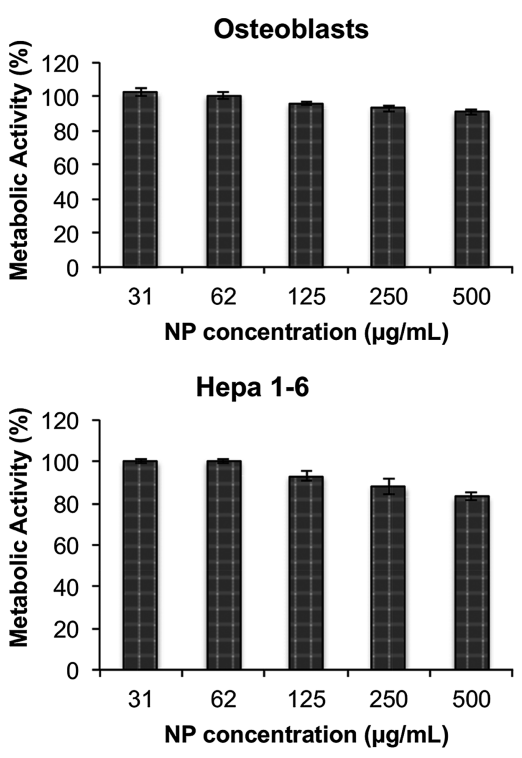

RAW 264.7

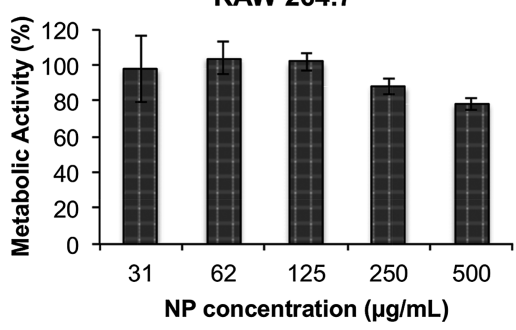

Figure 4. Cell viability after $24 \mathrm{~h}$ of incubation with increasing concentrations of Aln-PLGA NPs $(31-500 \mu \mathrm{g} / \mathrm{mL})$.

established fluorescent dye used for the visualization of intracellular and extracellular calcium. ${ }^{33}$ Fluorescent microscopy revealed that both post- and premodified NPs (imaged in the $750 \mathrm{~nm}$ channel) always co-localized with Quin-2 (480 $\mathrm{nm}$ ). However, as observed with the binding studies with HAp granules, Aln-PLGA NPs prepared by postmodification method showed a qualitatively higher binding to the mineral phases in comparison to that of NPs prepared by premodification with Aln (Figure 5). Pixel analysis confirmed an 8.4-fold increase in fluorescent signal (normalized to Quin-2 intensity, i.e., mineral phase) in the case of postmodified AlnPLGA NPs versus premodified NPs, suggesting that for a similar content of mineral phase, a greater number of NPs were associated in the case of the former. Because the amount of VT750 labeled PLGA was the same (5 wt \%) in both NP preparations, this observed enhancement of nearly 1 order of magnitude can be unequivocally attributed to the higher binding efficiency of the postmodified NPs to biogenic HAp.

USPIONSs Containing Aln-PLGA NPs Decrease $T_{2}$ Relaxation Times in Agarose Phantoms. Having demonstrated that Aln-PLGA NPs with encapsulated USPIONs possess favorable cytocompatibility and show high affinity for both synthetic and biogenic HAp, we ascertained the ability of this system to function as an MRI $T_{2}$-contrast agent. The system performance of NMR and MRI devices are usually tested with phantom systems. ${ }^{34,35}$ Agarose is one of the most suitable components available to fabricate imaging phantoms because it has comparable $T_{2}$ relaxation times compared with human tissue (40-150 ms). ${ }^{34}$ Hence, the Aln-PLGA NPs were first associated with HAp powder and then dispersed in agarose 

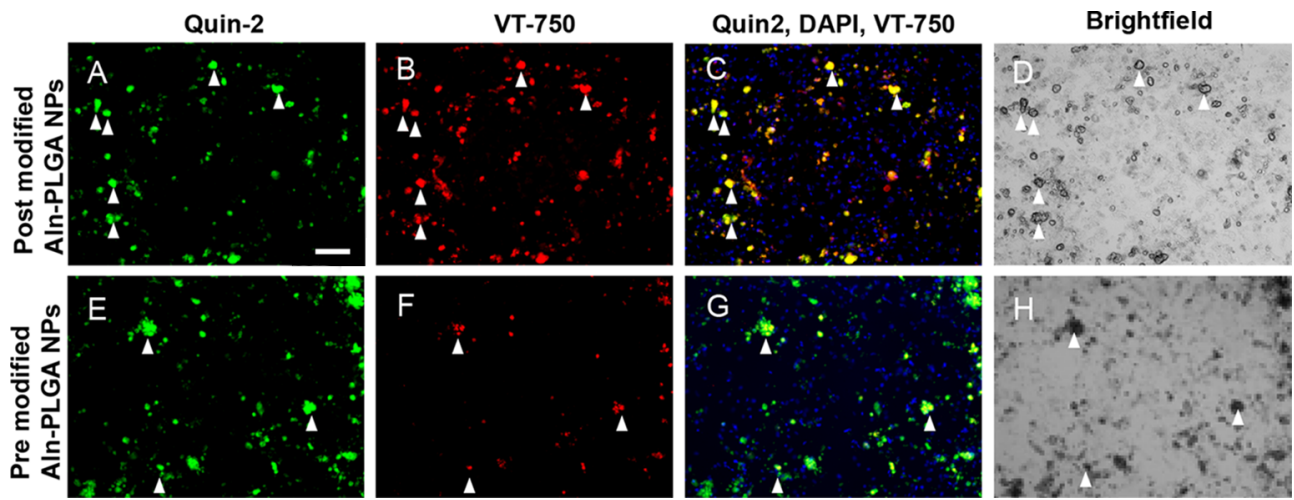

Figure 5. Binding of the PLGA NPs containing USPIONSs post- and premodified with Aln [Aln-PLGA (upper panel) and PLGA-Aln (lower panel), respectively] to biogenic HAp secreted by osteoblasts. The premodified NPs served as controls. (A and E) Quin-2 labeling of biogenic HAp deposits, (B and F) NPs, and (C and G) merged images showing colocalization of biogenic HAp (Quin-2) deposits with NPs (VT-750), and bluecolored cell nuclei (DAPI). Bright field images showing the mineral deposits (dark spots) associated with osteoblasts are shown in (D and H) for comparison. The scale bar is $10 \mu \mathrm{m}$.

A

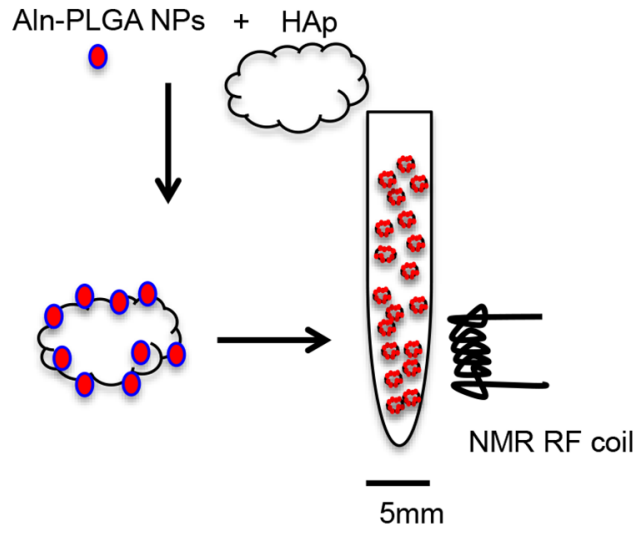

NMR Tube with gelated agarose and Aln-PLGA NPs on HAp
B

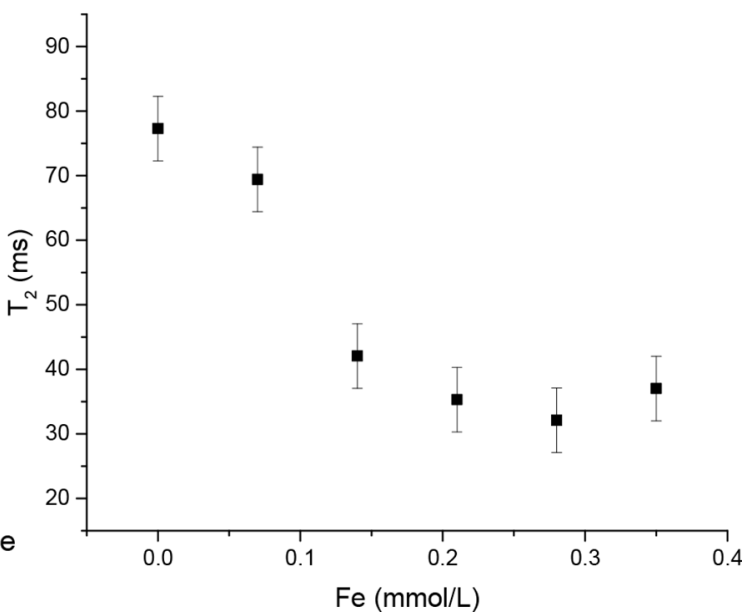

Figure 6. Water $T_{2}$ relaxation by the USPIONs encapsulated in the Aln-PLGA NPs: (A) schematic representation of experimental setup. (B) Water $T_{2}$ relaxation times in the presence of USPIONs of different concentrations within the NPs.

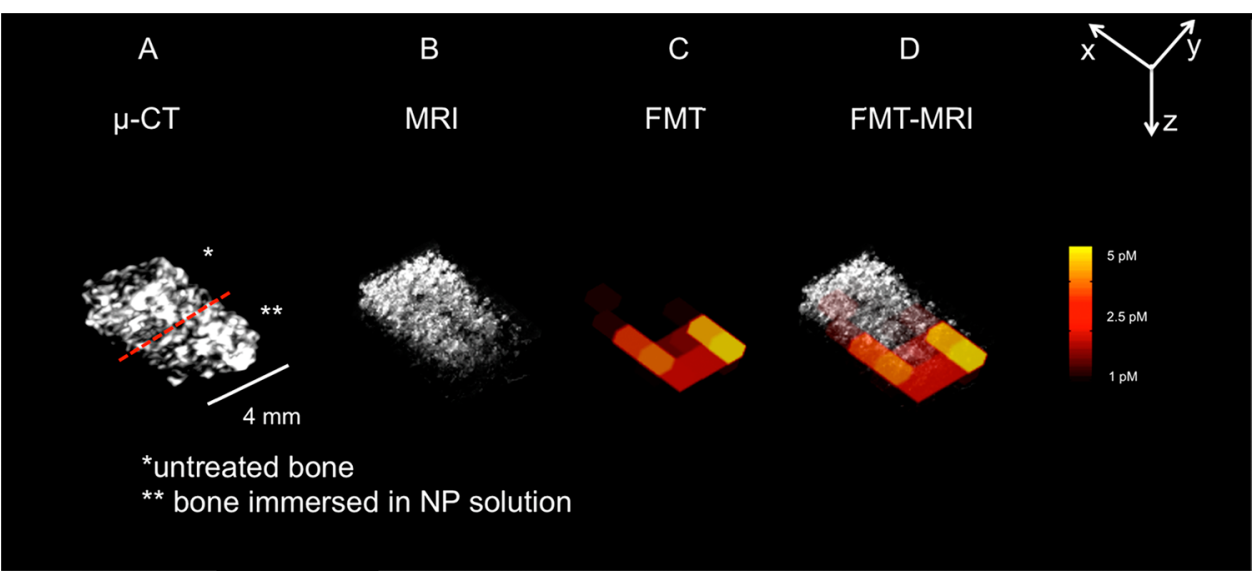

Figure 7. Imaging of fresh bovine femoral head spongy bone after incubation with the Aln-PLGA NPs labeled with VT750 and loaded with the USPIONs by $\mu$ CT (A), MRI (B), FMT (C), and MRI/FMT fused image (D). The dashed red line indicates the demarcation between the portion of the bone sample immersed in the NP solution to the bottom and the untreated region to the top. The color scale shows the concentration of NIR fluorophore VT750 in a given voxel $\left(1 \mathrm{~mm}^{3}\right)$.

gelled in an NMR tube to simulate bonelike tissue environment as shown in Figure 6A. The concentration of USPIONs in the
NPs was varied from 0.07 to $0.35 \mathrm{mmol} / \mathrm{L}$, and water $T_{2}$ relaxation times were measured using the Carr-Purcell- 
Meiboom-Gill (CPMG) pulse sequence. USPIONs encapsulated within the Aln-PLGA NPs affected the transverse $T_{2}$ relaxation as a function of the encapsulated amount of iron (Figure 6B). This clear inverse dependence of $T_{2}$ with concentration (see Figure $S 10$ for $R^{2}$ values and $r^{2}$ ) suggests that the Aln surface functionalization does not influence the ability of the encapsulated USPIONs to provide contrast. Even at a moderate concentration of $0.14 \mathrm{mmol} \mathrm{Fe} / \mathrm{L}$, a significant reduction in $T_{2}$ times of more than $50 \%$, that is, from 78 to 42 $\mathrm{ms}$, was observed with a further reduction to $\sim 35 \mathrm{~ms}$ at a concentration of $0.21 \mathrm{mmol} / \mathrm{L}$, which stagnated beyond 0.28 $\mathrm{mmol} / \mathrm{L}$. These findings confirm the suitability of Aln-PLGA NPs as the carrier for bone-targeted MRI contrast agents.

Aln-PLGA/VT750 Encapsulating USPIONs Enable Multimodal Imaging of Bone Environment in MR and NIR Optical Imaging. NIR probes have been successfully used to noninvasively gain information on biological processes. ${ }^{4,5}$ Combining the benefits of NIR imaging with the structural details gained from MRI can offer new means of correlating biological processes within dense structures such as bone. Having demonstrated that USPIONs encapsulated in Aln-PLGA NPs with high affinity toward biogenic HAp can decrease $T_{2}$ times of water, we investigated the potential of these novel nanoprobes to provide contrast in $T_{2}$-weighted MRI and NIR tomography using fresh bovine bone samples as a model system. Bovine bone samples (dimensions $4 \times 2 \times 6$ $\mathrm{mm}^{3}$ ) were immersed partially for $3 \mathrm{~h}$ in a solution of AlnPGLA NPs modified with VT750 and containing USPIONs. In this experimental design, the unimmersed half of the bone sample served as the internal control. The sample was washed four times with deionized water at $700 \mathrm{rpm}$ to remove unbound NPs and then imaged using $\mu$-computed tomography ( $\mu \mathrm{CT})$, MRI, and FMT (Figure 7). $\mu \mathrm{CT}$ can clearly distinguish spongy bone and was used to provide a reference point for the MRI and FMT image reconstruction. As postulated, a darkening in the MRI image was found exclusively in the region of the bone sample that was exposed to the Aln-PLGA NPs, thus confirming the ability of the NPs to not only bind to the bone tissue but also function as a $T_{2}$ contrast agent for MRI. The affinity of Aln-PLGA NPs to the bone was further verified using FMT, where a cumulative fluorescence of $32 \mathrm{pmol}$ of VT750 was found. This corresponds to a theoretical amount of $8.7 \mu \mathrm{g}$ of USPIONs. The ability to gain information in multiple modalities using these novel bone-targeting nanoprobes was verified by co-registration of the MRI and FMT data sets, where a clear overlap between the darkened regions (MRI modality) and the fluorescence signal (optical modality) was observed.

\section{CONCLUSIONS}

In this study, by combining a blending approach followed by postmodification with Aln, polymeric (PLGA) nanoprobes with bone-targeting properties were synthesized. The postmodification strategy enabled the encapsulation of USPIONs without interference from Aln. Furthermore, by introducing an NIRconjugated PLGA in the blending step, NPs possessing characteristics that can be interrogated in both MRI (USPIONs) and NIR optical tomography (NIR-dye) were realized for the first time. These novel multimodal nanoprobes show affinity to synthetic and biogenic HAp secreted by osteoblasts and found in spongy bone, and enable the interrogation of the bone tissue in both $T_{2}$-weighted MRI and optical modalities. The multimodal nanoprobes present an important platform to interrogate the bone environment to understand bone pathologies and develop bone-targeted therapeutics at an early stage.

\section{MATERIALS AND METHODS}

Materials. Hydroxyapatite (HAp) was purchased from Finceramica, Faenza (RA) Italy. Pro Osteon 200 was purchased from Zimmer Biomet in Winterthur, Zürich. Poly(D,L-lactideco-glycolide) (PLGA), $M_{\mathrm{w}}$ 14000-38000; poly(ethylene glycol) methyl ether-block-poly(lactide-co-glycolide) (PLGAPEG), PEG average $M_{\mathrm{n}}=5000 \mathrm{~g} / \mathrm{mol}$, PLGA average $M_{\mathrm{n}} 55$ $000 \mathrm{~g} / \mathrm{mol} ; \mathrm{N}$-(3-dimethylaminopropyl)- $N^{\prime}$-ethylcarbodiimide hydrochloride (EDC), $\geq 98 \%$; NHS, $98 \%$; aminubutyric acid, $\geq 98 \%$, phosphorus pentachloride, $\geq 98 \%$; ethylenediamine, $>99 \%$; methansulfonic acid, $\geq 98 \%$; sodium hydroxide, $\geq 98 \%$; dimethyl sulfoxide (DMSO), $\geq 99.8 \%$; Alizarin, dye content, 97\%; Quin-2, $\geq 95 \%$; dexamethasone, $\geq 98 \%$; L-ascorbic acid 2phosphate sesquimagnesium salt hydrate, $\geq 95 \%$; glycerol phosphate disodium salt pentahydrate, $\geq 98 \%$; and tetrahydrofuran (THF), $\geq 99.9 \%$ was purchased from Sigma-Aldrich, St. Louis, MO, USA. Methanol, $\geq 99.8 \%$ and agarose type I, of molecular-biological grade, was purchased from Merck Millipore, MA, USA. Magnesium sulfate, $\geq 99 \%$, and dialysis membranes [molecular weight cutoff (MWCO), 50000 and 10 000] were purchased from Carl Roth, Germany. Formaldehyde 37\% was purchased from AppliChem, Germany. Dichloromethane (DCM), $\geq 99 \%$, was purchased from Fischer Scientific, France. VivoTag-750 was purchased from Perkin Elmer, MA, USA. Acetone, $\geq 99.9 \%$ and diethylether, $\geq 99.9 \%$ was purchased from VWR International, PA, USA. Human marrow-derived MSCs were a gift from Prof. Dr. Ivan Martin from University Hospital Basel. Hepes buffer was purchased from PAN Biotech, Germany. Fetal bovine serum was purchased from Invitrogen, CA, USA. Penicillin-streptomycin and phosphate-buffered saline (PBS) were purchased form Gibco/Life Technologies, CA, USA. Fibroblast growth factor was purchased from R\&D system, Germany.

Synthesis of PLGA-NHS. Carboxylic acid end-functionalized PLGA $\left(2.9 \times 10^{-5} \mathrm{~mol}\right)$ was mixed with $\operatorname{EDC}(2.08 \times$ $10^{-4} \mathrm{~mol}$ ) in $10 \mathrm{~mL}$ DCM in an argon atmosphere. After 30 min, NHS $\left(2.08 \times 10^{-4} \mathrm{~mol}\right)$ was added to the reaction flask and stirred overnight. A translucent solution was obtained. The reaction mixture was filtered through a Teflon syringe filter with a cutoff of $200 \mathrm{~nm}$ and dripped into $10 \mathrm{~mL}$ of diethyl ether at $0{ }^{\circ} \mathrm{C}$ to form a precipitate. The obtained suspension was filtered, and the precipitate dried under reduced pressure to obtain PLGA-NHS.

VT-750 Functionalization of PLGA-NHS. PLGA- $\mathrm{NH}_{2}$ $(0.32 \mu \mathrm{mol})$ was diluted in $1 \mathrm{~mL}$ acetone. VivoTag-750 $(1 \mathrm{mg})$ was diluted in $100 \mu \mathrm{L}$ of DMSO and added to PLGA-NHS. The sample was put on a shaker for $3 \mathrm{~h}$ at $700 \mathrm{rpm}$. The acetone was removed under reduced pressure. Saturated $\mathrm{NaCl}$ $(0.4 \mathrm{~mL}), \mathrm{DCM}(2 \mathrm{~mL})$, and water $(2 \mathrm{~mL})$ were taken in a 5 $\mathrm{mL}$ Eppendorf tube, and the resultant mixture was shaken thoroughly. The water phase was removed, and the organic phase washed again with $2 \mathrm{~mL}$ of water. This step was repeated 5 times. The organic phase was dried with $\mathrm{MgSO}_{4}$, and the solvent evaporated under reduced pressure to yield PLGAVT750.

Synthesis of PLGA- $\mathrm{NH}_{2}$ from PLGA-NHS. PLGA-NHS $(100 \mathrm{mg}, 3.12 \mu \mathrm{mol})$ was diluted in $10 \mathrm{~mL}$ of DCM; and then 10 equiv of ethylenediamine was added to the solution and allowed to react at room temperature (RT) for $3 \mathrm{~h}$. The 
reaction mixture was precipitated in cooled diethyl ether, leading to a white precipitate, which was filtered and washed with diethyl ether. The filter cake was dried, dissolved in DCM, and reprecipitated in dried alcohol to remove unreacted amine.

Synthesis of Alendronic Acid. In a $250 \mathrm{~mL}$ flask under argon, amino butyric acid $(0.095 \mathrm{~mol})$ and phosphorous acid $(0.095 \mathrm{~mol})$ were dissolved while stirring at $65^{\circ} \mathrm{C}$ in $50 \mathrm{~mL}$ of methane sulfonic acid. After $20 \mathrm{~min}, 0.2 \mathrm{~mol}$ of $\mathrm{PCl}_{5}$ was added. The solution was stirred under reflux at $65{ }^{\circ} \mathrm{C}$ for $22 \mathrm{~h}$. The translucent mixture obtained was quenched with $200 \mathrm{~mL}$ of water under vigorous stirring. The formation of gas was observed, and the gas was absorbed with $\mathrm{NaOH} 0.1 \mathrm{~mol} / \mathrm{L}$. The reaction mixture was then stirred at reflux at $160{ }^{\circ} \mathrm{C}$ for 5 h. The reaction was cooled down using ice-cold water.

The $\mathrm{pH}$ in the initial reaction flask $(-0.34)$ was brought to 1.85 by the addition of $57 \mathrm{~mL}$ of $\mathrm{NaOH} 50 \%$ solution. The resulting solution was added dropwise to $500 \mathrm{~mL}$ of methanol, which was cooled with an ice bath. A white precipitate was formed. The suspension formed was filtered and washed with 3 $\times 100 \mathrm{~mL}$ of methanol. The resulting solid was solubilized in water $(30 \mathrm{~mL})$ and was poured over $500 \mathrm{~mL}$ of methanol at RT under vigorous agitation. The resulting white suspension was filtered to yield a white solid $(0.074 \mathrm{~mol})$. The remaining solvent was evaporated in the oven by heating for $1 \mathrm{~h}$ at $70^{\circ} \mathrm{C}$ followed by $20 \mathrm{~min}$ at $90{ }^{\circ} \mathrm{C}$.

Nanoprecipitation of NHS-PLGA NPs. The NPs were synthesized using the nanoprecipitation method. ${ }^{31}$ Briefly, PLGA-VT750, PLGA-PEG, and PLGA-NHS (5:15:80 w/ $\mathrm{w} \%)$ were dissolved in THF $(1 \mathrm{~mL})$ and USPIONs (0.1 $\mathrm{mmol} / \mathrm{L})$ at a fixed mass ratio with a concentration of $5 \mathrm{mg} /$ $\mathrm{mL}$. To the organic phase an equal amount of water was added rapidly to form the NPs. The remaining organic solvent was removed by dialysis $(\mathrm{MWCO}=50000 / 50 \mathrm{kDa})$, stirring at 200 rpm overnight at RT.

Alendronic Acid Conjugation on the Surface of NHSPLGA NPs. Alendronic acid $(500 \mu \mathrm{L}, 0.2 \mathrm{mg} / \mathrm{mL})$ was mixed with $500 \mu \mathrm{L}$ of NHS-PLGA NPs. The sample was shaken for 2 $\mathrm{h}$ at $700 \mathrm{rpm}$ at RT. To remove the unreacted alendronic acid, the sample was dialyzed overnight against water (MWCO 100 $000 \mathrm{kDa}$ ).

Synthesis of PLGA-Aln from PLGA-NHS. PLGA-NHS $(10 \mathrm{mg}, 0.32 \mu \mathrm{mol})$ was dissolved in $5 \mathrm{~mL}$ of acetone. Water $(5$ $\mathrm{mL}$ ) was slowly added while stirred with a magnetic stirrer. A milky suspension of NHS-PLGA NPs was obtained. The solvent was removed by dialysis $(\mathrm{MWCO}=50000 / 50 \mathrm{kDa}$ ) overnight. The NHS-PLGA NPs were diluted with water to reach a concentration of $0.25 \mathrm{mg} / \mathrm{mL}$. Alendronic acid $(1 \mathrm{~mL}$; $0.1 \mathrm{mg} / \mathrm{mL}$ ) was added dropwise to the NP suspension while stirring. The NPs started to aggregate, indicating the formation of an amide bond between alendronic acid and PLGA-NHS. The reaction was stirred for $2 \mathrm{~h}$ at RT. The precipitate was centrifuged (2000 rpm), and the supernatant was discharged. The precipitate was dissolved in DCM and again precipitated in diethyl ether. To remove the unreacted alendronic acid, the sample was again dialyzed overnight against water (MWCO $100000 \mathrm{kDa}$ ), which yielded PLGA-Aln. The resulting PLGA-Aln was used for the control experiments.

Cell Culture. Hepa 1-6 cells were cultured in Dulbecco's Modified Eagle's Medium supplemented with 10\% fetal bovine serum (Thermo Fisher Scientific, Waltham, MA, USA) and 1\% penicillin/streptomycin (P/S) $(100 \mathrm{U} / \mathrm{mL}$; PAN-Biotech $\mathrm{GmbH}$, Aidenbach, Germany). Human MSCs, a gift from Prof. Ivan Martin (University Hospital Basel Reference
Number of local ethical committee 78/07), were differentiated into osteoblasts for 21 days with modified eagle's medium $(\alpha$ MEM) supplemented with $10 \%$ fetal bovine serum, $100 \mathrm{U} / \mathrm{mL}$ penicillin, $100 \mu \mathrm{g} / \mathrm{mL}$ streptomycin, $1 \mathrm{mM}$ sodium pyruvate, 10 $\mathrm{mM}$ HEPES buffer, $5 \mathrm{ng} / \mathrm{mL}$ basic fibroblast growth factor, 10 $\mathrm{nM}$ dexamethasone, $0.1 \mathrm{mM}$ L-ascorbic acid-2-phosphate, and $10 \mathrm{mM} \beta$-glycerol phosphate. Osteoblast differentiation was tested after 7 days with RT-qPCR and after 21 days with Alizarin Red-S staining. Mouse macrophages (RAW 267.4, Sigma Aldrich, Germany) were cultured in a Roswell Park Memorial Institute medium supplemented with $5 \%$ fetal bovine serum and $1 \% \mathrm{P} / \mathrm{S}(100 \mathrm{U} / \mathrm{mL}$; PAN-Biotech $\mathrm{GmbH}$, Aidenbach, Germany). All cells were maintained in a humidified incubator at $37{ }^{\circ} \mathrm{C}$ with $5 \% \mathrm{CO}_{2}$ atmosphere.

Cellular Metabolic Activity Assessment. MTT is an assay used to investigate the mitochondrial activity of cells. Cells were exposed to the MTT dye (3-(4,5-dimethylthiazol-2yl)-2,5-diphenyltetrazolium bromide), which transforms into insoluble purple formazan crystals. After exposure, the crystals were dissolved in DMSO, and the absorbance at $530 \mathrm{~nm}$ was measured in a plate reader (Synergy II, BioTek Instruments, Inc.). The amount of transformed MTT is proportional to the metabolic activity and can therefore be used to determine the effect of particles on the cell viability. We seeded $20 \times 10^{3}$ cells per well in a 96-well plate and allowed them to attach overnight. On the following day, the supernatant was replaced with media containing Aln-PLGA NPs in different concentrations $(31,62,125,250$, and $500 \mu \mathrm{g} / \mathrm{mL})$. After $24 \mathrm{~h}$ of incubation, cell viability was investigated with the protocol provided by the supplier (Invitrogen). Untreated cells served as controls for the experiment.

Osteogenic Differentiation of Human MSCs. MSCs were differentiated to osteoblasts for 21 days with $\alpha$-MEM supplemented with $10 \%$ fetal bovine serum, $100 \mathrm{U} / \mathrm{mL}$ penicillin, $100 \mu \mathrm{g} / \mathrm{mL}$ streptomycin, $1 \mathrm{mM}$ sodium pyruvate, $10 \mathrm{mM}$ HEPES buffer, $5 \mathrm{ng} / \mathrm{mL}$ basic fibroblast growth factor, $10 \mathrm{nM}$ dexamethasone, $0.1 \mathrm{mM}$ L-ascorbic acid-2-phosphate, and $10 \mathrm{mM} \beta$-glycerol-phosphate. Osteoblast differentiation was tested after 7 days with RT-qPCR and after 21 days with Alizarin Red-S staining.

Alizarin Red-S Staining. After three weeks, the cell layer in the petri dish was washed with PBS for 3 times, fixed for $10 \mathrm{~min}$ in $3.7 \%$ formalin at RT, stained with Alizarin Red 2\% for 20 min, and washed with tap water. Human MSCs were able to mineralize the matrix abundantly after 3 weeks of culture in osteogenic medium, as demonstrated by a strong Alizarin Red-S staining.

Transmission Electron Microscopy. To determine the size and the presence of USPIONs in the polymeric NPs TEM was used [Zeiss LEO 912 Omega (Leo Elektronenmikroskopie $\mathrm{GmbH}$, Oberkochen, Germany)]. An aliquot of Aln-PLGA NPs in water was placed on a carbon grid. After removing the solvent, the samples were analyzed using an electron acceleration of $120 \mathrm{keV}$.

Scanning Electron Microscopy. SEM micrographs were obtained using a Quanta 250 FEG (FEI). The dried HAp samples (Finceramica) after incubation with the AlnPLGANPs were placed on a carbon grid and coated with gold to visualize under high-resolution under reduced pressure. For elemental analysis (EDX), an Oxford INCA x-act (Oxford Instruments, UK) was used. The samples were analyzed using the software INCA. 
Fourier-Transform Infrared Spectroscopy (FTIR). FTIR spectra were recorded on a Vector 22 instrument (Bruker Optics), and the software provided by the manufacturer was used to import and analyze the spectrum.

Dynamic Light Scattering. The particle size was analyzed using dynamic light scattering with a Delsa Nano C (Beckman Coulter Inc., USA) provided with a laser diode operating at 658 $\mathrm{nm}$. Measurements were conducted at a scattering angle of $\theta=$ $165^{\circ}$ to the incident beam. Samples were equilibrated at $25^{\circ} \mathrm{C}$ for at least $30 \mathrm{~min}$ prior to the analysis. The data were processed using CONTIN algorithms with Delsa Nano UI Software version 3.73. The size and PDI were expressed as the average of at least three measurements ( \pm standard deviation).

Surface Charge Measurements. A Delsa Nano C (Beckman Coulter Inc., USA) analyzer was used to measure $\zeta$ potential values. The $\zeta$ potential of the particles in aqueous suspension ( $1: 9$ dilution with deionized $\mathrm{H}_{2} \mathrm{O}$ ) was obtained by measuring the electrophoretic movement of charged particles under an applied electric field. Scattered light was detected at a $30^{\circ}$ angle at $25^{\circ} \mathrm{C}$.

$T_{2}$ Measurements. A Bruker Avance 300 nuclear magnetic resonance spectrometer (Bruker Biosciences Corp., Billerica, MA) with a CPMG sequence was used to measure $T_{2}$ relaxation times. The USPIONs containing the Aln NPs were incubated with $20 \mathrm{mg}$ HAp powder for $3 \mathrm{~h}$ at RT. The supernatant was removed, and the samples were washed 5 times with $0.2 \mathrm{~mL}$ of double-distilled deionized water. The samples were then dried under reduced pressure for $6 \mathrm{~h}$. A $2 \%$ $\mathrm{w} / \mathrm{v}$ agarose solution prepared in deionized water was heated to $90{ }^{\circ} \mathrm{C}$ to form a solution, and while cooling it down, $20 \mathrm{mg}$ HAp mixed with $0.7 \mathrm{~mL}$ of agarose was added to the solution in an NMR-tube $\left(180 \times 5 \mathrm{~mm}^{2}\right)$, following which the suspension was gelled by placing it on ice. The NMR data were processed using Top Spin 3.2 software (Bruker), and Dynamic Center 2.1.8 was used to calculate $T_{2}$ relaxation times.

Microscopy. For fluorescence microscopy, cells were seeded in 8-well chamber slides. Following incubation of the osteoblasts with Aln-PLGA NPs for $2 \mathrm{~h}$, the cells were washed three times with PBS to remove unbound and weakly bound NPs, stained with 4',6-diamidino-2-phenylindole (DAPI) nuclear stain, fixed with paraformaldehyde 3.7\% (v/v) for 15 min, mounted onto coverslips, and then imaged using the Zeiss cell observer Z1. The images of the NPs (750 channel) were exported and analyzed using pixel analysis from ImageJ to compare the amount of NPs (pre- and postmodified) bound on the calcium deposits.

FMT Imaging. After incubation with fluorescence-labeled NPs, the Hap (Osteoporon) was dried under reduced pressure and sealed in a plastic tube $3.0 \times 18.0 \mathrm{~mm}^{2}$, which was placed in an agarose phantom ( $1 \%$ in Milli-Q water). Samples were analyzed with an FMT 2500 (Perkin-Elmer) using the $750 \mathrm{~nm}$ channel (excitation $745 \mathrm{~nm}$, emission 770-800 nm). Reflectance images were analyzed using a TrueQuant 3D (Perkin-Elmer, version 2.2.0.24).

Image Co-registration. $\mu \mathrm{CT}$ volumes (isometric voxel size: $0.0844 \mu \mathrm{m} ; 908 \times 428$ pixel, $500-1200$ slices) and FMT volumes were imported into AMIDE (64-bit v. 1.0.4). The volumes were corrected for axial rotations and co-registered based on the reflectance images from FMT. The co-registered datasets were exported in DICOM format and imported into Osirix (v. 5.5.1, 64-bit), where the image volumes were synchronized, fused, and visualized using $3 \mathrm{D}$ volume rendering.
MRI. A commercially available mousehead 2-element quadrature cryogenic coil system with a $7 \mathrm{~T}$ Bruker BioSpec small horizontal animal scanner (bore size $20 \mathrm{~cm}$, maximum gradient amplitude $676 \mathrm{mT} / \mathrm{m}$ ) was used to adapt MR pulse sequences with respect to bone sample imaging. The standard setup, designed for imaging live mice, was modified by the addition of a 3D printed sample holder made of polylactic acid (PLA). Bone samples incubated with NPs (spongiosa, $4 \times 2 \times$ $6 \mathrm{~mm}^{3}$ ) were then inserted into a poly(methyl methacrylate) (PMMA) container (height $3 \mathrm{~mm}$ ) filled with saline solution and sealed on one side with an adhesive PCR tape. For imaging, a 3D FLASH sequence was applied with TR (40 ms), TE (6.2 $\mathrm{ms})$, flip angle $\left(50^{\circ}\right)$, averages (1), BW $(50000 \mathrm{~Hz})$, Cryocoil, pulse length $(1.400 \mathrm{~ms}), \mathrm{BW}(3000.0 \mathrm{~Hz})$ and a physical resolution of $39 \times 38 \times 94 \mu \mathrm{m}^{3}$, and the imaging was obtained in $22 \mathrm{~min}$. The binding of the NPs on the bone samples was subsequently compared with the findings in FMT microscopy and $\mu \mathrm{CT}$.

$\boldsymbol{\mu C T}$ Imaging. Bovine bone samples were scanned using a SkyScan 1178 (SkyScan, Belgium; version 1.3) high-speed in vivo micro-CT imager over an angle of $180^{\circ}$ with rotation steps of $1^{\circ}$ at a resolution of $83 \mu \mathrm{m}$ and a field of view of $86 \mathrm{~mm}$. Each image was acquired at $65 \mathrm{kV}$ and $615 \mu \mathrm{A}$ with an average of three frames. Using the NRecon module (SkyScan 2010, v. 6.3.3), the data sets underwent postalignment, beam-hardening correction, and ring-artifact reduction (a parameter set with the integrated fine-tuning tool), and the $3 \mathrm{D}$ reconstructions were exported in a DICOM format and presented as a $3 \mathrm{D}$ image in OsiriX v. 5.5.1.

Statistical Analysis. The statistical analysis was performed using Student's $t$-test (paired, type 2, and 2-tailed) in Microsoft Excel, and $p$-values $<0.05$ were considered statistically significant. All values are presented as mean \pm standard deviation $(\mathrm{SD})$.

\section{ASSOCIATED CONTENT}

\section{Supporting Information}

The Supporting Information is available free of charge on the ACS Publications website at DOI: 10.1021/acsomega.6b00088. TEM of premodified Aln-PLGA NPs (from PLGAAln) containing USPIONs; evaluation of PLGA-PEG weight-percentage to yield stable NPs; stability of AlnPLGA/PEG-PLGA NPs in serum-conditioned media; reaction scheme and structure analysis of alendronic acid; EDX spectra of NHS-PLGA NPs; ${ }^{31} \mathrm{P}$ spectra of NHSPLGA NPs; SEM images of Aln-PLGA NPs adsorption on HAp over time; SEM images of pristine HAp, AlnPLGA NPs incubated in cell-conditioned media; PLGAPEG NPs; osteogenic lineage of osteoblasts; Alizarin Red staining of calcium deposits secreted by osteoblasts; and $R^{2}$ values and $r^{2}$ as a function of USPION concentration in Aln-PLGA NPs (PDF)

\section{AUTHOR INFORMATION}

\section{Corresponding Author}

*E-mail: prasad.shastri@gmail.com. Phone: +49-761-203-6268. Fax: +49 761-203-5016.

\section{Notes}

The authors declare no competing financial interest. 


\section{ACKNOWLEDGMENTS}

This work was funded by the Fifth INTERREG Upper Rhine Program (Project A21: NANO@MATRIX), the Excellence Initiative of the German Federal and State Governments (Grant EXC 294, Centre for Biological Signalling Studies), the Helmholtz Zentrum Geesthacht through the Helmholtz Virtual Institute on Multifunctional Biomaterials for Medicine, and the University of Freiburg. The authors thank R. Thomann for assistance with acquiring TEM images, V. Ahmadi for SEM analysis, D. Vonwil for technical advice on FMT, J. Christensen for valuable input in image co-registration, S. Shah for assistance with FMT imaging of HAp samples, V. Hugo Pacheco Torres for assistance with NMR measurements, and $\mathrm{K}$. Göbel-Guéniot for preparing the sample holder for the MRI measurements.

\section{REFERENCES}

(1) Ratzinger, G.; Agrawal, P.; Körner, W.; Lonkai, J.; Sanders, H. M. H. F.; Terreno, E.; Wirth, M.; Strijkers, G. J.; Nicolay, K.; Gabor, F. Surface modification of PLGA nanospheres with Gd-DTPA and GdDOTA for high-relaxivity MRI contrast agents. Biomaterials 2010, 31, 8716-8723.

(2) Gallo, J.; Kamaly, N.; Lavdas, I.; Stevens, E.; Nguyen, Q.-D.; Wylezinska-Arridge, M.; Aboagye, E. O.; Long, N. J. CXCR4-Targeted and MMP-Responsive Iron Oxide Nanoparticles for Enhanced Magnetic Resonance Imaging. Angew. Chem., Int. Ed. 2014, 53, 9550-9554.

(3) Christensen, J.; Vonwil, D.; Shastri, V. P. Non-Invasive In Vivo Imaging and Quantification of Tumor Growth and Metastasis in Rats Using Cells Expressing Far-Red Fluorescence Protein. PLoS One 2015, 10, No. e0132725.

(4) Weissleder, R.; Ntziachristos, V. Shedding light onto live molecular targets. Nat. Med. 2003, 9, 123-128.

(5) Nahrendorf, M.; Waterman, P.; Thurber, G.; Groves, K.; Rajopadhye, M.; Panizzi, P.; Marinelli, B.; Aikawa, E.; Pittet, M. J.; Swirski, F. K.; Weissleder, R. Hybrid in vivo FMT-CT imaging of protease activity in atherosclerosis with customized nanosensors. Arterioscler., Thromb., Vasc. Biol. 2009, 29, 1444-1451.

(6) Zaheer, A.; Lenkinski, R. E.; Mahmood, A.; Jones, A. G.; Cantley, L. C.; Frangioni, J. V. In vivo near-infrared fluorescence imaging of osteoblastic activity. Nat. Biotechnol. 2001, 19, 1148-1154.

(7) Vonwil, D.; Christensen, J.; Fischer, S.; Ronneberger, O.; Shastri, V. P. Validation of Fluorescence Molecular Tomography/Micro-CT Multimodal Imaging In Vivo in Rats. Mol. Imaging Biol. 2014, 16, 350-361.

(8) Harada, S.-I.; Rodan, G. A. Control of osteoblast function and regulation of bone mass. Nature 2003, 423, 349-355.

(9) Shea, J.; Miller, S. Skeletal function and structure: Implications for tissue-targeted therapeutics. Adv. Drug Delivery Rev. 2005, 57, 945957.

(10) Mundy, G. R. Metastasis to bone: Causes, consequences and therapeutic opportunities. Nat. Rev. Cancer 2002, 2, 584-593.

(11) Jung, A.; Bisaz, S.; Fleisch, H. The Binding of Pyrophosphate and Two Diphosphonates by Hydroxyapatite Crystals. Calcif. Tissue Res. 1973, 11, 269-280.

(12) Swami, A.; Reagan, M. R.; Basto, P.; Mishima, Y.; Kamaly, N.; Glavey, S.; Zhang, S.; Moschetta, M.; Seevaratnam, D.; Zhang, Y.; Liu, J.; Memarzadeh, M.; Wu, J.; Manier, S.; Shi, J.; Bertrand, N.; Lu, Z. N.; Nagano, K.; Baron, R.; Sacco, A.; Roccaro, A. M.; Farokhzad, O. C.; Ghobrial, I. M. Engineered nanomedicine for myeloma and bone microenvironment targeting. Proc. Natl. Acad. Sci. U.S.A. 2014, 111, 10287-10292.

(13) Wang, D.; Miller, S.; Sima, M.; Kopečková, P.; Kopeček, J. Synthesis and evaluation of water-soluble polymeric bone-targeted drug delivery systems. Bioconjugate Chem. 2003, 14, 853-859.
(14) Clementi, C.; Miller, K.; Mero, A.; Satchi-Fainaro, R.; Pasut, G. Dendritic poly(ethylene glycol) bearing paclitaxel and alendronate for targeting bone neoplasms. Mol. Pharmaceutics 2011, 8, 1063-1072.

(15) Low, S. A.; Kopeček, J. Targeting polymer therapeutics to bone. Adv. Drug Delivery Rev. 2012, 64, 1189-1204.

(16) Vachal, P.; Hale, J. J.; Lu, Z.; Streckfuss, E. C.; Mills, S. G.; MacCoss, M.; Yin, D. H.; Algayer, K.; Manser, K.; Kesisoglou, F.; Ghosh, S.; Alani, L. L. Synthesis and study of alendronate derivatives as potential prodrugs of alendronate sodium for the treatment of low bone density and osteoporosis. J. Med. Chem. 2006, 49, 3060-3063.

(17) de Rosales, R. T. M.; Tavaré, R.; Glaria, A.; Varma, G.; Protti, A.; Blower, P. J. ${ }^{99 \mathrm{~m}} \mathrm{Tc}$-bisphosphonate-iron oxide nanoparticle conjugates for dual-modality biomedical imaging. Bioconjugate Chem. 2011, 22, 455-465.

(18) Sandiford, L.; Phinikaridou, A.; Protti, A.; Meszaros, L. K.; Cui, X.; Yan, Y.; Frodsham, G.; Williamson, P. A.; Gaddum, N.; Botnar, R. M.; Blower, P. J.; Green, M. A.; de Rosales, R. T. M. Bisphosphonateanchored PEGylation and radiolabeling of superparamagnetic iron oxide: Long-circulating nanoparticles for in vivo multimodal (T1 MRISPECT) imaging. ACS Nano 2013, 7, 500-512.

(19) Cole, L. E.; Vargo-Gogola, T.; Roeder, R. K. Targeted delivery to bone and mineral deposits using bisphosphonate ligands. Adv. Drug Delivery Rev. 2016, 99, 12-27.

(20) Liess, C.; Lüsse, S.; Karger, N.; Heller, M.; Glüer, C.-C. Detection of changes in cartilage water content using MRI $T_{2}$-mapping in vivo. Osteoarthritis Cartilage 2002, 10, 907-913.

(21) Boskey, A. L. Bone composition: Relationship to bone fragility and antiosteoporotic drug effects. BoneKEy Rep. 2013, 2, 1-11.

(22) Pope, J. M.; Repin, N. A Simple Approach to $T_{2}$ Imaging in MRI. Magn. Reson. Imaging 1988, 6, 641-646.

(23) Laurent, S.; Mahmoudi, M. Superparamagnetic iron oxide nanoparticles: Promises for diagnosis and treatment of cancer. Int. J. Mol. Epidemiol. Genet. 2011, 2, 367-390.

(24) Lalatonne, Y.; Monteil, M.; Jouni, H.; Serfaty, J. M.; SainteCatherine, O.; Lièvre, N.; Kusmia, S.; Weinmann, P.; Lecouvey, M.; Motte, L. Superparamagnetic Bifunctional Bisphosphonates Nanoparticles: A Potential MRI Contrast Agent for Osteoporosis Therapy and Diagnostic. J. Osteoporosis 2010, 2010, 747852.

(25) Reddy, L. H.; Arias, J. L.; Nicolas, J.; Couvreur, P. Magnetic nanoparticles: Design and characterization, toxicity and biocompatibility, pharmaceutical and biomedical applications. Chem. Rev. 2012, $112,5818-5878$

(26) Wyss, P. P.; Lamichhane, S.; Rauber, M.; Thomann, R.; Krämer, K. W.; Shastri, V. P. Tripod USPIONs with high aspect ratio show enhanced $T_{2}$ relaxation and cytocompatibility. Nanomedicine 2016, 11, $1017-1030$.

(27) Amstad, E.; Gillich, T.; Bilecka, I.; Textor, M.; Reimhult, E. Ultrastable iron oxide nanoparticle colloidal suspensions using dispersants with catechol-derived anchor groups. Nano Lett. 2009, 9, 4042-4048.

(28) Danhier, F.; Lecouturier, N.; Vroman, B.; Jérôme, C.; Marchand-Brynaert, J.; Feron, O.; Préat, V. Paclitaxel-loaded PEGylated PLGA-based nanoparticles: In vitro and in vivo evaluation. J. Controlled Release 2009, 133, 11-17.

(29) Danhier, F.; Vroman, B.; Lecouturier, N.; Crokart, N.; Pourcelle, V.; Freichels, H.; Jérôme, C.; Marchand-Brynaert, J.; Feron, O.; Préat, V. Targeting of tumor endothelium by RGD-grafted PLGA-nanoparticles loaded with paclitaxel. J. Controlled Release 2009, 140, 166173

(30) Choi, S.-W.; Kim, J.-H. Design of surface-modified poly(D,Llactide-co-glycolide) nanoparticles for targeted drug delivery to bone. J. Controlled Release 2007, 122, 24-30.

(31) Sussman, E. M.; Clarke, M. B., Jr.; Shastri, V. P. Single-step process to produce surface-functionalized polymeric nanoparticles. Langmuir 2007, 23, 12275-12279.

(32) Inman, M.; Visconti, A.; Yan, C.; Siegel, D.; Ross, D.; Moody, C. $\mathrm{J}$. Antitumour indolequinones: Synthesis and activity against human pancreatic cancer cells. Org. Biomol. Chem. 2014, 12, 4848-4861. 
(33) Takahashi, A.; Camacho, P.; Lechleiter, J. D.; Herman, B. Measurement of intracellular calcium. Physiol. Rev. 1999, 79, 10891125.

(34) Mitchell, M. D.; Kundel, H. L.; Axel, L.; Joseph, P. M. Agarose as a tissue equivalent phantom material for NMR imaging. Magn. Reson. Imaging 1986, 4, 263-266.

(35) Hellerbach, A.; Schuster, V.; Jansen, A.; Sommer, J. MRI Phantoms-Are There Alternatives to Agar? PLoS One 2013, 8, No. e70343. 